\title{
Is it Time That We Start Using Sonovisible Catheters in Regional Anesthesia?
}

\section{Neisevilie Nisa*}

Department of Anesthesia, All India Institute of Medical Sciences, Delhi, India

\section{Case History}

The growth of ultrasound (US) technology has popularized thoracic paravertebral blocks (PVB) due to its ability to visualize the paravertebral space and the surrounding structures like pleura. Intrathoracic placement of a paravertebral catheter has rarely been reported under US guidance [1]. We had a case of inadvertent intrathoracic catheter placement during US guided PVB.

A 45 year old male, ASA physical status 1 weighing $67 \mathrm{~kg}$ and height measuring $162 \mathrm{~cm}\left(\mathrm{BMI}=24.1 \mathrm{~kg} / \mathrm{m}^{2}\right)$ was scheduled for right upper lobectomy. In the operating room (OR), routine ASA monitors were attached and patient was sedated with intravenous midazolam and fentanyl and supplemental oxygen was delivered via facemask. The patient was placed in the left lateral position for the PV catheter placement.

An initial US scan of the targeted paravertebral region (T3-T6) was performed with a portable US, SonoSite 180 plus and a linear array $6-13 \mathrm{MHz}$ probe (SonoSite Drive SE Bothell, WA USA). The US probe was aligned in a longitudinal paramedian plane between two transverse processes. The transverse processes with the wedge shape PV space and the lung parenchyma sliding beneath the pleura were easily visualized. After skin and subcutaneous tissue anesthesia, an 18 gauge $5 \mathrm{~cm}$ long Tuohy needle (StimuLong Plus Plexus Catheter, Pajunk, Germany) was introduced in plane to the caudal end of the probe.

The tip of the needle was advanced under direct visualization until it pierced the superior cost transverse ligament and was lying immediately above the pleura. The needle tip location was verified and negative aspiration of blood or air was confirmed. After verifying the location of the needle tip, $10 \mathrm{~mL}$ of $0.25 \%$ bupivacaine was injected and the spread of local anesthetic (LA) with depression of the pleura was clearly visualized.

Following LA administration a 20 gauge catheter was inserted beyond the tip of the needle in the PV space using normal saline for hydro-dissection and the catheter was threaded without difficulty. During catheter advancement there was no resistance and the patient had no pain or discomfort or symptoms of pleural irritation and the procedure went uneventful. After general anesthesia induction, the patient was positioned in the left lateral decubitus position and surgery was started. Midway during the surgery, a bolus dose of $10 \mathrm{~mL}$ of $0.25 \%$ bupivacaine was given. The surgeon noticed the drug seeping into the surgical field. The surgical field was mopped and the catheter was found just penetrating the parietal pleura (Figure 1). There was no injury to the lung parenchyma. The catheter was withdrawn by $1 \mathrm{~cm}$ under visualization and was further used for perioperative analgesia without any complications.

The risk of pneumothorax due to accidental pleural puncture with PVB is $0.5 \%$ [2] and US technology has further decreased this rate. However, US alone will not eliminate all complications of regional techniques. In contrast to the easy puncture of the paravertebral space, the catheter insertion is more difficult. The most probable cause of misplaced catheters is due to over-threading of the catheters through the needle [3]. Currently, there are no specially designed PV catheters

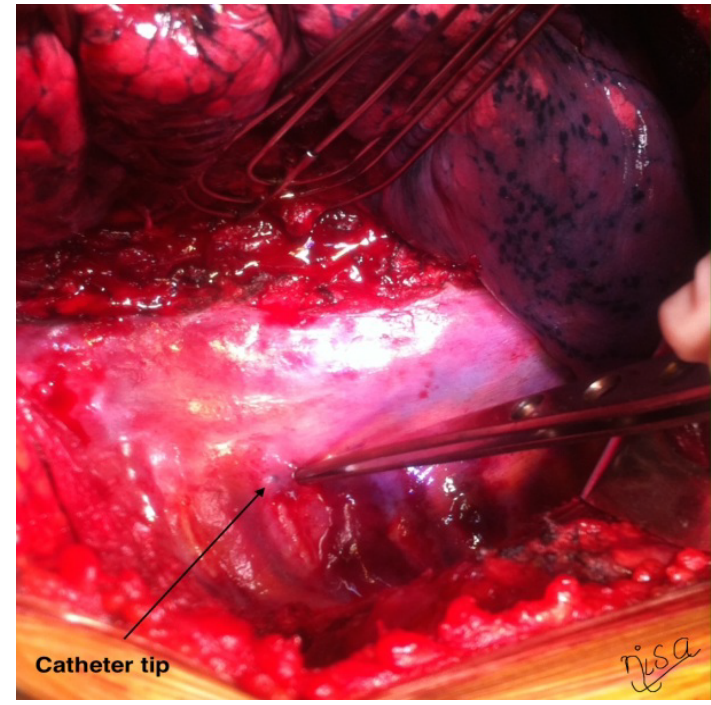

Figure 1: Sonovisible catheter penetrating the parietal pleura during surgery.

which aids in easy threading and thus the process of threading the catheters still remains a blind procedure after the needle tip is positioned in the PV space under US guidance. There are no randomized control trial upto date which compares the visibility of the different catheters available in market during US guided regional anesthesia, and future studies are warranted in this matter. The visualization of the catheters does not depend upon the width of the catheters but the material used which impacts on the reflectivity of the US beam that enhance the dark at the center of the catheter and improve its visualization [4]. The brightest images are obtained when the ultrasound beam reflects perpendicular to the catheter. This may hold true for improving needle visualization but less fruitful when applied to catheters as it is difficult to practically attain such alignment.

PVB are used for other surgeries on the chest wall like fibroadenoma breast, mastectomy, gynecomastia etc where the chest wall is not opened. In such cases, pleural puncture like the one in our case can cause pneumothorax and other complications for breaching the pleural cavity like intrapleural injection of LA and anesthesia of the diaphragm. The role of the surgeon inserting the PVB catheter intraoperatively is feasible in thoracotomy but not in such cases like breast surgeries. Thus,

*Corresponding author: Neisevilie Nisa, Department of Anesthesia, All India Institute of Medical Sciences, Delhi, India, Tel: 08376960134; E-mail: neisevilie@ gmail.com

Received May 13, 2016; Accepted June 10, 2016; Published June 17, 2016

Citation:Nisa N (2016) Is it Time That We Start Using Sonovisible Catheters in Regional Anesthesia?. Gen Med (Los Angel) 4: 250. doi:10.4172/23275146.1000250

Copyright: (c) 2016 Nisa N. This is an open-access article distributed under the terms of the Creative Commons Attribution License, which permits unrestricted use, distribution, and reproduction in any medium, provided the original author and source are credited. 
Citation: Nisa N (2016) Is it Time That We Start Using Sonovisible Catheters in Regional Anesthesia?. Gen Med (Los Angel) 4: 250. doi:10.4172/23275146.1000250

there is a strong argument for visualization of the catheters during threading under US guidance that they remain within the paravertebral space to prevent complications like pneumothorax.

With the growing popularity of thoracic PVB and the risk of pleural puncture in hand while threading catheters blindly, is time that we start using sonovisible catheters in regional anesthesia.

\section{References}

1. Yavuz G, Asli GA, Mine S (2012) Pleural puncture and intrathoracic catheter placement during ultrasound guided paravertebral block. J Cardiothorac Vasc Anesthesia 10: 1053.

2. Naja MZ, Lonnqvist PA (2001) Somatic paravertebral nerve blockade: incidence of failed block and complications. Anaesthesia 56: 1184 -1188.

3. Luyet C, Herrmann G, Ross S (2011) Ultrasound-guided thoracic paravertebral puncture and placement of catheters in human cadavers: where do catheters go? British Journal of Anesthesia 106: 246-254.

4. Junji T, Naozumi T, Kentaro O (2012) Ultrasound visibility of regional anesthesia catheters: an in vitro study. Korean J Anesthesiol 63: 59-64. 